Discussion Paper No. 898

\title{
PAIRWISE PARTITION GRAPHS \\ AND \\ STRATEGY-PROOF SOCIAL CHOICE \\ IN THE EXOGENOUS \\ INDIFFERENCE CLASS MODEL
}

\author{
Anup Pramanik \\ Arunava Sen
}

April 2014

The Institute of Social and Economic Research Osaka University

6-1 Mihogaoka, Ibaraki, Osaka 567-0047, Japan 


\title{
Pairwise Partition Graphs AND Strategy-proof Social Choice in the Exogenous Indifference Class Model
}

\author{
Anup Pramanik *and Arunava Sen ${ }^{\dagger}$
}

April 14, 2014

\begin{abstract}
In this paper we consider the exogenous indifference classes model of Barberá and Ehlers (2011) and Sato (2009) and analyze further the relationship between the structure of indifference classes across agents and dictatorship results. The key to our approach is the pairwise partition graph. We provide necessary conditions on these graphs for strategy-proofness and unanimity (or efficiency) to imply dictatorship. These conditions are not sufficient; we also provide separate stronger conditions that are sufficient. A full characterization is obtained in the case of two agents for domains where strategy-proofness and efficiency imply dictatorship.
\end{abstract}

Keywords And Phrases: Social choice functions, Strategy-proofness, Dictatorial Domains, Exogenous Indifference Class, Gibbard- Satterthwaite theorem.

JEL Classification Number: D71.

${ }^{*}$ Institute of Social and Economic Research, Osaka University, 6-1, Mihogaoka, Ibaraki, 567-0047, Japan. E-mail: anup@iser.osaka-u.ac.jp

†Indian Statistical Institute, New Delhi, India. 


\section{INTRODUCTION}

The seminal work of Gibbard (1977) and Satterthwaite (1975) showed that all deterministic strategy-proof SCFs with a range of at least three alternatives and defined over the complete domain, is dictatorial. A large body of literature has since focused on relaxing the underlying assumptions of this result. A natural way to do this is to impose domain restrictions. Indeed, many real life problems have inherent domain restrictions. This paper is a contribution to this stand of literature.

It is well known that the structure of strategy-proof SCFs becomes more complex when indifference is permitted in individual preferences. ${ }^{1}$ In this paper, we investigate a model of domain restrictions involving indifference, the exogenous indifference class model first introduced in Barberá and Ehlers (2011). In this model, the indifference classes of agents' preferences is exogenously given. In particular, every individual has an exogenous partition of the set of alternatives. An individual is always indifferent between alternatives $a$ and $b$ iff both $a$ and $b$ belong to the same element of her partition set. But an individual's ranking of the different elements of her partition set, is complete.

This framework includes several well-studied models as special cases. For instance, the case of private goods and selfish preferences is one where an individual is indifferent between all alternatives that give her the same commodity bundle. It includes the one-sided matching model studied in Svensson (1999),Pápai (2000). It also includes the Gibbard-Satterthwaite framework where the elements of the partition are all singletons. Further examples are provided in Sato (2009).

The goal of Barberá and Ehlers (2011) was to study the Arrovain aggregation issue in the exogenous indifference class model. Sato (2009) examined the same model from the perspective of strategic voting. This is an interesting model in this respect as well because it covers both the complete domain over which strategy-proofness implies dictatorship (the Gibbard-Sattherwaite Theorem) as well as the private good allocation model for which it is well-known that a rich class of strategy-proof social choice functions exist (Pápai (2000)). Sato showed that the number of common indifference classes is critical to the existence of strategy-proof and non-dictatorial SCFs. He assumed that common indifference classes are singletons and obtained two results. First, an onto and strategy-proof social choice function is dictatorial whenever there are at least three common indifference classes. Second, the same result holds when the number of common indifference classes is two, provided that unanimity is strengthened to efficiency.

In this paper, we further examine the relationship between dictatorship results in this model and the structure of indifference classes across agents. Our results are formulated in terms of the pairwise partition graph induced by the indifference classes. Fix a pair of agents $i$ and $j$ and their indifference classes. The partition graph for this pair is a bipartite graph

\footnotetext{
${ }^{1}$ See for instance, the literature of strategy-proofness in classical exchange economies originating from Hurwicz (1972) and Satterthwaite and Sonnenschein (1981).
} 
whose vertices are $i$ and $j$ 's indifference classes. There are no edges between the vertices representing the indifference classes of a given agent; vertices for $i$ and $j$ ' have an edge if the indifference classes representing these vertices have no common alternative. We show that a necessary condition for strategy-proofness and unanimity to imply dictatorship in the domain induced by a partition is that each associated pairwise partition graph is connected with the degree of every vertex being at least two. This requirement can be weakened to the graphs being connected (with possibly isolated vertices), if unanimity is replaced by efficiency.

We are unfortunately, unable to show that these necessary conditions are sufficient for dictatorship. However we are able to identify a number of stronger conditions that are sufficient. The first of these is the existence of at least two common indifference classes with no restrictions on their size - a result which clearly generalizes that of Sato. In addition we have three sufficient conditions for the case of two voters. One applies to the case where there is exactly one common indifference class while another shows that strategy-proofness and unanimity imply dictatorship when the partition graph is a cycle. Finally, we show that with the stronger assumption of efficiency, strategy-proofness implies dictatorship when the partition graph is connected with possibly isolated vertices. The last condition implies that we have a necessary and sufficient condition for dictatorship for the case of efficiency when there are exactly two voters.

We now proceed to details.

\section{The Model}

Let $A=\{a, b, c, \ldots\}$ denote a finite set of alternatives with $|A|=m$. Let $I=\{1, \ldots, n\}$, $n \geq 2$ be a finite set of agents. We impose restrictions on the domain of preferences following Barberá and Ehlers (2011). Each agent $i$ has a partition $S_{i}$ of $A$ that is exogenously specified and independent of preferences. A typical element of $S_{i}$ is $s_{i}^{j}$ where $j=1, \ldots J$. An ordering $R\left(S_{i}\right)$ over $A$ respects $S_{i}$ if (i) a pair of alternatives belonging to the same element of $S_{i}$ are indifferent to each other and (ii) otherwise one is strictly preferred to the other. Let $I\left(S_{i}\right)$ and $P\left(S_{i}\right)$ denote the symmetric and assymetric components of $R\left(S_{i}\right)$ respectively. Let $\mathbb{R}\left(S_{i}\right)$ be the set of all orderings respecting $S_{i}$. Let $S=\left(S_{1}, \ldots, S_{n}\right)$ be an $n$-tuple of partitions, one for each agent. The admissible preference domain is $\mathbb{R}(S)=\prod_{i \in N} \mathbb{R}\left(S_{i}\right)$.

OBSERVATION 1 For a given partition $S_{i}, \mathbb{R}\left(S_{i}\right)$ consists of all possible strict orderings over the elements of $S_{i}$. If all agents have the same partition, the model reduces to the standard voting model where the elements of the partition can be thought of as an alternative. The essence of the problem is that partitions across agents can differ. Suitable choices of $S_{i}$ 's yield the usual private goods allocation model with selfish preferences, the universal domain with strict orderings as well as several interesting intermediate cases as shown in Sato (2009). 
Example 1 Let $A=\{a, b, c, d, e\}$ and $I=\{1,2\}$. Let $S_{1}=\{a b, c, d e\}$ and $S_{2}=\{a b, c d, e\}$. Note that Table 1 and Table 2 below represent $\mathbb{R}\left(S_{1}\right)$ and $\mathbb{R}\left(S_{2}\right)$ respectively.

\begin{tabular}{|c|c|c|c|c|c|c|c|c|c|}
\hline$R_{1}^{2}$ & $R_{1}^{3}$ & $R_{1}^{4}$ & $R_{1}^{5}$ & $R_{1}^{6}$ & $R_{2}^{1}$ & $R_{2}^{2}$ & $R_{2}^{3}$ & $R_{2}^{4}$ & $R_{2}^{5}$ \\
\hline$a b$ & $c$ & $c$ & $d e$ & $d e$ & $a b$ & $a b$ & $c d$ & $c d$ & $e$ \\
\hline$d e$ & $a b$ & $d e$ & $a b$ & $c$ & $c d$ & $e$ & $a b$ & $e$ & $a b$ \\
\hline$c$ & $d e$ & $a b$ & $c$ & $a b$ & $e$ & $c d$ & $e$ & $a b$ & $c d$ \\
\hline
\end{tabular}

Table 1: $\mathbb{R}\left(S_{1}\right)$

Table 2: $\mathbb{R}\left(S_{2}\right)$

Whenever $S$ or $S_{i}$ is fixed, $\mathbb{R}(S), R(S), \mathbb{R}\left(S_{i}\right)$, and $R\left(S_{i}\right)$ will be simply written as $\mathbb{R}, R$, $\mathbb{R}_{i}$, and $R_{i}$ respectively. Fix a partition $S_{i}$. An indifference class of agent $i$ is an element of $S_{i}$. For any $R_{i} \in \mathbb{R}_{i}$ and $k \in\{1, \ldots, J\}$, the $k^{t h}$ ranked indifference class in $R_{i}$ is denoted by $r_{k}\left(R_{i}\right)$ (in other words, there are $k-1$ elements of $S_{i}$ ranked strictly higher than $r_{k}\left(R_{i}\right)$ ). For any $D \subseteq A$ and $R_{i} \in \mathbb{R}_{i}, M\left(R_{i}, B\right)$ is the set of maximal elements in $B$ according to $R_{i}$ i.e. $M\left(R_{i}, B\right)=\left\{a \in B: a R_{i} b\right.$ for all $\left.b \in B\right\}$.

Let $V(S)=\bigcap_{i=1}^{n} S_{i}$ denote the set of common indifference classes for the partition $n$-tuple $S$. Also let $v(S)=|V(S)|$ denote the number of common indifference classes. Note that in Example 1, $V(S)=\{a b\}$ and $v(S)=1$. Moreover, in the private goods allocation model with selfish preferences $v(S)=0$ while in the standard voting model $v(S)=m$.

Definition 1 A social choice function (SCF) is a mapping $f: \mathbb{R} \rightarrow A$.

Each agent's preference ordering is private information, i.e. known only to herself. These preferences must therefore be elicited by the mechanism designer. If a SCF is strategy-proof, then no agent can benefit by misrepresenting her preferences irrespective of her beliefs about the preference announcement of other agent.

Definition $2 A S C F f: \mathbb{R} \rightarrow A$ is manipulable by agent $i$ at a profile $R \in \mathbb{R}$ via $R_{i}^{\prime}$ if

$$
f\left(R_{i}^{\prime}, R_{-i}\right) P_{i} f(R)
$$

A SCF $f$ is strategy-proof if it is not manipulable by any agent at any profile.

The following additional properties of SCFs are standard.

Definition $3 A S C F f$ is unanimous if for all $R \in \mathbb{R}$

$$
f(R) \in \bigcap_{i=1}^{n} r_{1}\left(R_{i}\right) \text { whenever } \bigcap_{i=1}^{n} r_{1}\left(R_{i}\right) \neq \emptyset .
$$

A unanimous SCF always respects consensus whenever it exists, i.e. if all agents agree on some set of alternatives as their best, then the SCF must pick an element from this set. A stronger condition than unanimity is efficiency. 
Definition $4 A S C F f$ is efficient if for all $R \in \mathbb{R}$, for all $a, b \in A$

$$
\left[\begin{array}{c}
a R_{i} b \text { for all } i \in I \\
a P_{i} b \text { for some } j \in I
\end{array}\right] \Rightarrow[f(R) \neq b]
$$

If agents preferences are strict but sufficiently "rich", strategy-proofness and unanimity implies efficiency. In our setting where indifference is permitted, this proposition typically does not hold. We therefore consider the consequences of unanimity and efficiency separately.

Definition 5 A SCF $f$ is dictatorial if there exists an agent $i$ such that $f(R) \in r_{1}\left(R_{i}\right)$ for all $R \in \mathbb{R}$.

An important feature of our model is that a SCF is not fully specified even when an agent is a dictator since this agent will typically have more than one best alternative at any profile.

An important subclass of dictatorial SCFs are serial dictatorships which we define below.

Definition 6 A priority $\sigma$ is a one-to-one map $\sigma: I \rightarrow I$. Let $R$ be a profile and define the sets $T_{\sigma(1)}(R), T_{\sigma(2)}(R), \ldots, T_{\sigma(n)}(R)$ inductively as follows:

$$
\begin{aligned}
T_{\sigma(1)}(R) & =M\left(R_{\sigma(1)}, A\right) \\
T_{\sigma(2)}(R) & =M\left(R_{\sigma(2)}, T_{\sigma(1)}(R)\right) \\
& \vdots \\
T_{\sigma(n)}(R) & =M\left(R_{\sigma(n)}, T_{\sigma(n-1)}(R)\right)
\end{aligned}
$$

A serial dictatorship with respect to $\sigma$ satisfies $f^{\sigma}(R) \in T_{\sigma(n)}(R)$ for all $R$.

A priority is a linear arrangement of agents where $\sigma(1)$ is the first agent, $\sigma(2)$ the second and so on with $\sigma(n)$ being the last. A serial dictatorship (with respect to $\sigma$ ) works as follows: at any profile $R$, agent $\sigma(1)$ picks her maximal elements in $A, \sigma(2)$ picks her maximal elements in $\sigma(1)$ 's maximal elements and so on. Note that a serial dictatorship is not fully specified because $T_{\sigma(n)}(R)$ may contain more than one alternative.

\section{Results}

Our goal is to investigate the structure of indifference classes across agents for which unanimity (or efficiency) and strategy-proofness imply dictatorship. Our first step is to provide necessary conditions for dictatorship. Subsequently, we provide various sufficient conditions. 


\subsection{Necessary Conditions for Dictatorship}

These condition will be formulated in terms of graphs arising from the set $S$. We briefly review some basic graph-theoretic concepts.

A Graph $G$ is a pair of finite sets $V$ and $E$ where $V$ is the set of vertices or nodes and $E$ is the set of edges. An edge is a non-ordered pair of vertices. If $e=\{u, v\}$ is an edge, i.e. $e \in E$, then $u$ and $v$ are adjacent vertices and $u$ and $e$ are incident as are $v$ and $e$. The degree of a vertex $v, \operatorname{deg}_{G}(v)$ is the number of edges incident with $v$. An isolated vertex is a vertex with degree zero. A path $u v$ is a finite sequence of distinct vertices $\left(v_{1}, v_{2}, \ldots, v_{k}\right)$ where $v_{1}=u, v_{k}=v$ and $\left\{v_{i}, v_{i+1}\right\} \in E$ for all $i \in\{1, \ldots, k-1\}$. If the path $u v$ exist, then $u$ and $v$ are connected. A graph is connected if all pairs of distinct vertices are connected. A graph is discrete if $E=\{\emptyset\}$. A graph is a connected graph with isolated vertices if (i) it is not discrete and (ii) the sub-graph consisting of non-isolated vertices is connected. A graph is bipartite if the vertices of $G$ can be partitioned in to two subsets $V_{1}$ and $V_{2}$ in such a way that no two vertices in the same subset have an edge.

Let $i, j$ be agents and $S_{i}$ and $S_{j}$ be partitions for $i$ and $j$ respectively. The graph $G\left(S_{i}, S_{j}\right)$ is constructed as follows:

(i) The set $V=V_{1} \cup V_{2}$ where $V_{1}=S_{i}$ and $V_{2}=S_{j}$.

(ii) There are no edges between vertices in $V_{1}$ or between vertices in $V_{2}$.

(iii) There is an edge between $s_{i}^{l}$ and $s_{j}^{k}$ iff $s_{i}^{l} \cap s_{j}^{k} \neq \emptyset$ for all $s_{i}^{l} \in S_{i}$ and $s_{j}^{k} \in S_{j}$.

The graph $G\left(S_{i}, S_{j}\right)$ is bipartite. We refer to it as the Partition Graph for $i$ and $j$. We provide several examples of such graphs below.

Example 2 Let $I=\{j, i\}, A=\{a, b, c, d, e, f, g, h\}, \bar{S}_{j}=\{a b, c d, e f, g h\}$ and $\bar{S}_{i}=$ $\{a c, b d, e g, f h\}$. The induced partition Graph is shown in Figure 1 and it is not connected. There is no path between $g h$ to $f h$. Also every vertex of it has degree 2 .

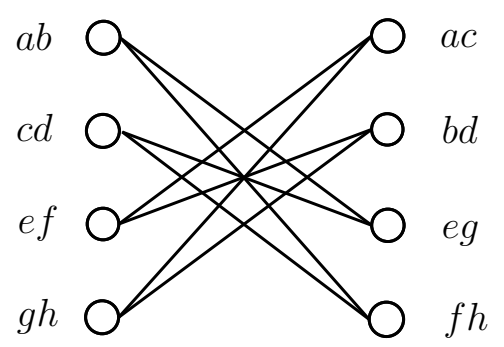

Figure 1: Disconnected graph

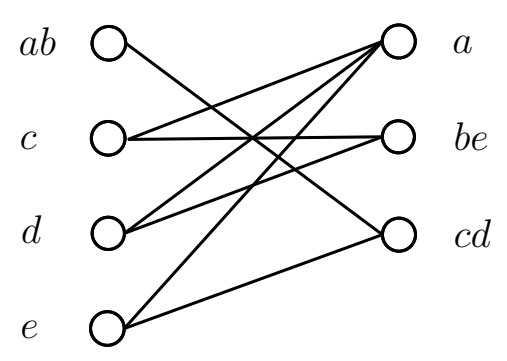

Figure 2: A graph with a degree one vertex 
ExAmple 3 Let $I=\{j, i\}, A=\{a, b, c, d, e\}, \hat{S}_{j}=\{a b, c, d, e\}$ and $\hat{S}_{i}=\{a, b e, c d\}$. Figure 2 shows the induced partition graph which is connected. Note that degree of the vertex $a b$ is 1 .

ExAmple 4 Let $I=\{j, i\}, A=\{a, b, c, d, e\}, \tilde{S}_{j}=\{a b, c, d, e\}$ and $\tilde{S}_{i}=\{a, b e, c d\}$. The induced partition graph is shown in Figure 3 which is not connected. Note that degree of the vertex $a b c$ is 0 .

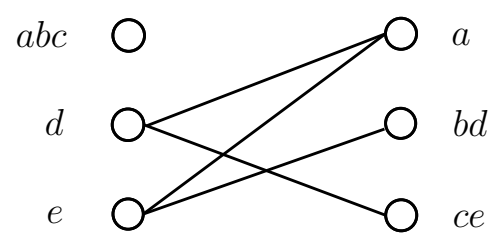

Figure 3: A graph with an isolated vertex
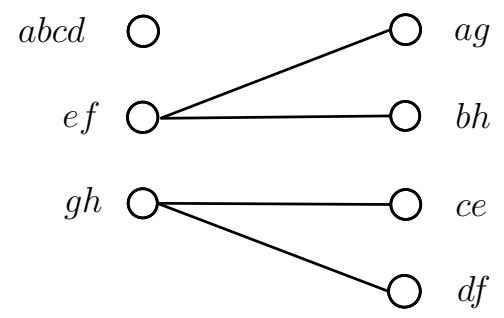

Figure 4: A non-connected graph with isolated vertices

ExAmple 5 Let $I=\{j, i\}, A=\{a, b, c, d, e, f, g, h\}, S_{j}^{o}=\{a b c d, e f, g h\}$ and $S_{i}^{o}=$ $\{a g, b h, c e, d f\}$. The induced partition graph is shown in Figure 4 and it is not connected. Moreover it is not a connected graph with isolated vertices.

Example 6 (The Allocation Problem with Selfish Preferences) There are three objects, say houses $a, b$ and $c$ which have to be allocated among three agents. An allocation is an ordered triple such as $a b c$ where the first, second and third components refer to the houses allocated to agents 1,2 and 3 respectively. The set of allocations is the set $A=$ $\{a b c, a c b, b a c, b c a, c a b, c b a\}$. Consider the standard model of selfish preferences. The induced partition $S^{*}=\left(S_{1}^{*}, S_{2}^{*}, S_{3}^{*}\right)$ is as follows:

- $S_{1}^{*}=\{\{a b c, a c b\},\{b a c, b c a\},\{c a b, c b a\}\}$.

- $S_{2}^{*}=\{\{a b c, c b a\},\{b a c, c a b\},\{b c a, a c b\}\}$.

- $S_{3}^{*}=\{\{a b c, b a c\},\{c b a, b c a\},\{c a b, a c b\}\}$.

The Partition Graph $G\left(S_{1}^{*}, S_{2}^{*}\right)$ is shown in Figure 5. Note that there are no common indifference classes. Clearly $G\left(S_{1}^{*}, S_{2}^{*}\right)$ is not connected, for instance there is no path between $\{c a b, c b a\}$ and $\{b c a, a c b\}$.

ExAmple 7 Let $I=\{j, i\}, A=\{a, b, c, d, e, f, g, h, i\}, S_{j}^{\prime}=\{a b c$, def, ghi $\}$ and $S_{i}^{\prime}=$ $\{a d g, b e h, c f i\}$. The induced partition graph in Figure 6, is a discrete graph. 


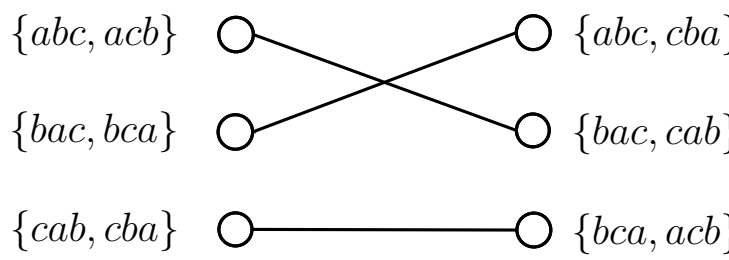

Figure 5: $G\left(S_{1}^{*}, S_{2}^{*}\right)$

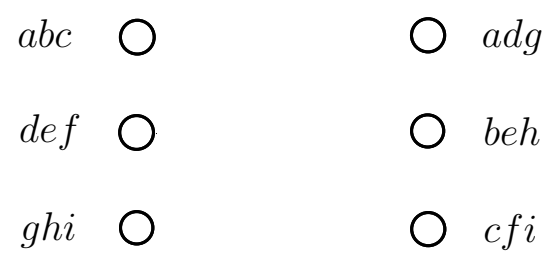

Figure 6: Discrete graph

Observation 2 Suppose $I=\{i, j\}$ and $S$ is such that $G\left(S_{i}, S_{j}\right)$ is discrete (Example 7). Then every unanimous SCF is dictatorial. Therefore the discrete partition graph case is trivial when there are two agents and can be excluded from consideration. In our necessary conditions (Theorem 1 below), we assume that the partition graph is not discrete for all pairs of agents. Note that if there are three or more agents, the pairwise discreteness of all partition graphs does not imply that every unanimous SCF is dictatorial. We are unable to provide an answer for the case where partition graphs are pairwise discrete (or even some of them are pairwise discrete) but unanimity does not imply dictatorship.

Theorem 1 Let $S=\left(S_{1}, \ldots, S_{n}\right)$ be such that $G\left(S_{i}, S_{j}\right)$ is not discrete for all $i, j \in I$.

A. Suppose $[f: \mathbb{R} \rightarrow A$ is strategy-proof and unanimous $\Rightarrow f$ is dictarorial $]$. Then $G\left(S_{i}, S_{j}\right)$ is connected and the degree of every vertex is at least 2 for all $i, j \in I$.

B. Suppose $[f: \mathbb{R} \rightarrow A$ is strategy-proof and efficient $\Rightarrow f$ is dictarorial $]$. Then $G\left(S_{i}, S_{j}\right)$ is a connected graph with isolated vertices for all $i, j \in I$.

Proof: We first prove Part A. Pick an arbitrary pair $i, j \in I$. We consider three cases regarding $G\left(S_{i}, S_{j}\right)$ : (I) it is not connected but has no isolated vertices (such as Example 2) (II) it is connected and has a vertex with degree 1 (Example 3) and (III) it has an isolated vertex (Example 4). These cases cover all possible cases in the statement of $\mathrm{A}$ and we show that in each case it is possible to construct a unanimous, strategy-proof and non-dictatorial $\mathrm{SCF} f: \mathbb{R} \rightarrow A$.

Case I: The vertices of $G\left(S_{i}, S_{j}\right)$ can be partitioned into subsets $V^{\prime}$ and $V^{\prime \prime}$ with $\left|V^{\prime}\right|$, $\left|V^{\prime \prime}\right| \geq 2$ and $\{u, v\} \notin E$ for all $u \in V^{\prime}$ and $v \in V^{\prime \prime}$ (In Figure 2, $V^{\prime}$ is the set $\{a b, c d, e g, f h\}$ and $V^{\prime \prime}$ is the set $\left.\{a c, b d, e f, g h\}\right)$. We fix two priority functions $\sigma_{1}$ and $\sigma_{2}$ as follows: $\sigma_{1}(1)=i, \sigma_{1}(2)=j, \sigma_{2}(1)=j, \sigma_{2}(2)=i$ and $\sigma_{1}(k)=\sigma_{2}(k)$ for all $k \neq 1,2$. Let $\mathbb{D}$ be the set of preference profiles such that $r_{1}\left(R_{i}\right), r_{1}\left(R_{j}\right) \in V^{\prime}$. The SCF will be a serial dictatorship with priority $\sigma_{1}$ in the domain $\mathbb{D}$ and $\sigma_{2}$ elsewhere, i.e.

$$
f(R)= \begin{cases}\text { a serial dictatorship with respect to the priority function } \sigma_{1} & \text { if } R \in \mathbb{D} \\ \text { a serial dictatorship with respect to the priority function } \sigma_{2} & \text { if } R \notin \mathbb{D}\end{cases}
$$

We first check the strategy-proofness of $f$. Observe that an agent $k \neq i, j$ cannot manipulate because of the nature of serial dictatorships and the fact that they cannot affect 
their priority. Consider a profile $R \in \mathbb{D}$. The outcome is a $R_{j}$-maximal element outcome in the first-ranked indifference class of $i$ in $R_{i}$, say $s_{i}^{1}$. Since $i$ is a dictator, she does not manipulate. Clearly $j$ cannot manipulate via an ordering $R_{j}^{\prime}$ such that $\left(R_{i}, R_{j}^{\prime}, \ldots\right) \in \mathbb{D}$ because $i$ will remain the dictator. Suppose $j$ attempts to manipulate via an ordering $R_{j}^{\prime}$ such that $\left(R_{i}, R_{j}^{\prime}, \ldots\right) \notin \mathbb{D}$. He is now dictator and the outcome belongs to $r_{1}\left(R_{j}^{\prime}\right)$. However, $r_{1}\left(R_{j}^{\prime}\right) \cap s_{i}^{1} \neq \emptyset$ by properties of the sets $V^{\prime}$ and $V^{\prime \prime}$; the outcome is therefore belongs to $s_{i}^{1}$. Hence $j$ cannot manipulate. The only remaining case to consider is a possible manipulation by $i$ from a profile not belonging to $\mathbb{D}$ to a profile in $\mathbb{D}$. The argument to show that $i$ cannot gain is identical to the case where $j$ attempts to manipulate from $\mathbb{D}$ to a profile outside it.

Unanimity is satisfied because $f$ is a serial dictatorship at every profile. There are profiles in $\mathbb{D}$ and outside it where $i$ and $j$ 's best ranked alternatives have an empty interesection. Therefore, neither $i$ nor $j$ are dictators. If $l \neq i, j$ is a dictator, then $G\left(S_{i}, S_{l}\right)$ and $G\left(S_{j}, G_{l}\right)$ are discrete, contradicting our assumption- i.e. $f$ is non-dictatorial.

Case II: Let $u$ be a vertex with degree 1. Assume w.l.o.g that $u \in S_{j}$ and $u=s_{j}^{k}$. By assumption, there exists a (unique) vertex $s_{i}^{m} \in S_{i}$ such that $\left\{s_{i}^{m}, s_{j}^{k}\right\} \in E$ (For instance in Example $3, s_{j}^{k}$ and $s_{i}^{m}$ are $a b$ and $c d$ respectively). Let $\mathbb{D}$ be the set of preference profiles such that $r_{1}\left(R_{i}\right)=s_{i}^{m}$. We fix a priority function $\sigma$ such that $\sigma(1)=j$ and $\sigma(2)=i$. Let $R \in \mathbb{D}$ and define the sets $T_{\sigma(1)}^{*}(R), T_{\sigma(2)}^{*}(R), \ldots, T_{\sigma(n)}^{*}(R)$ inductively as follows:

$$
\begin{aligned}
T_{\sigma(1)}^{*}(R) & =M\left(R_{\sigma(1)}, s_{j}^{k} \cup s_{i}^{m}\right) \\
T_{\sigma(2)}^{*}(R) & =M\left(R_{\sigma(2)}, T_{\sigma(1)}^{*}\right) \\
& \vdots \\
T_{\sigma(n)}^{*}(R) & =M\left(R_{\sigma(n)}, T_{\sigma(n-1)}^{*}(R)\right)
\end{aligned}
$$

We define a $\mathrm{SCF} f_{1}^{\sigma}: \mathbb{D} \rightarrow A$ as follows: $f_{1}^{\sigma}(R) \in T_{\sigma(n)}^{*}(R)$ for all $R \in \mathbb{D}$.

Let $\bar{\sigma}$ be a priority function such that $\bar{\sigma}(1)=i, \bar{\sigma}(2)=j$ and $\bar{\sigma}(k)=\sigma(k)$ for all $k \neq 1,2$. We define the following $\mathrm{SCF} f: \mathbb{R} \rightarrow A$ :

$$
f(R)= \begin{cases}f_{1}^{\sigma}(R) & \text { if } R \in \mathbb{D} \\ \text { a serial dictatorship with respect to the priority function } \bar{\sigma} & \text { if } R \notin \mathbb{D}\end{cases}
$$

In order to check the strategy-proofness of $f$, it suffices to check that $i$ and $j$ cannot manipulate. Consider $R \in \mathbb{D}$ - the outcome is a $R_{j}$-maximal element in $s_{j}^{k} \cup s_{i}^{m}$. Agent $j$ cannot manipulate since $\left(R_{i}, R_{j}^{\prime} \ldots\right) \in \mathbb{D}$ for all $R_{j}^{\prime}$. If the outcome belongs to $s_{i}^{m}$ then clearly $i$ cannot manipulate. Otherwise, the outcome belongs to $s_{j}^{k}$. Since $s_{j}^{k}$ is a vertex with degree one, it has a non-empty intersection with every indifference class other than $s_{i}^{m}$. Hence the outcome belongs to $r_{2}\left(R_{i}\right)$. By construction, $i$ cannot obtain an alternative in $r_{1}\left(R_{i}\right)=s_{i}^{m}$. If $R \notin \mathbb{D}$, agent $i$ is dictator and cannot manipulate. Note that $j$ cannot manipulate at $R \notin \mathbb{D}$ because $\left(R_{j}^{\prime}, R_{i}, \ldots\right) \notin \mathbb{D}$ for all $R_{j}^{\prime}$. 
Unanimity and non-dictatorship of $f$ is easily verified.

Case III: Let $u$ be a vertex with degree 0. Assume w.l.o.g that $u \in S_{j}$ and $u=s_{j}^{k}$. Since $G\left(S_{i}, S_{j}\right)$ is not discrete, there exists an edge $\left\{s_{i}^{m}, s_{j}^{l}\right\}$ where $s_{j}^{k} \neq s_{j}^{l}$ (In Figure 3, the node $s_{j}^{k}$ and the edge $\left\{s_{i}^{m}, s_{j}^{l}\right\}$ are $a b c$ and $\{d, a\}$ respectively). Let $\mathbb{D}$ be the set of preference profiles where $r_{1}\left(R_{i}\right)=s_{i}^{m}, r_{1}\left(R_{j}\right)=s_{j}^{l}$ and $r_{2}\left(R_{j}\right)=s_{j}^{k}$. We fix a priority function $\sigma$ such that $\sigma(1)=j$ and $\sigma(2)=i$. Let $R \in \mathbb{D}$ and define the sets $T_{\sigma(1)}^{*}(R), T_{\sigma(2)}^{*}(R), \ldots, T_{\sigma(n)}^{*}(R)$ inductively as follows:

$$
\begin{aligned}
T_{\sigma(1)}^{*}(R) & =s_{j}^{k} \\
T_{\sigma(2)}^{*}(R) & =r_{2}\left(R_{\sigma(2)}\right) \cap s_{j}^{k} \\
T_{\sigma(3)}^{*}(R) & =M\left(R_{\sigma(3)}, T_{\sigma(2)}^{*}(R)\right) \\
& \vdots \\
T_{\sigma(n)}^{*}(R) & =M\left(R_{\sigma(n)}, T_{\sigma(n-1)}^{*}(R)\right)
\end{aligned}
$$

We define a SCF $f_{1}^{\sigma}: \mathbb{D} \rightarrow A$ as follows: $f_{1}^{\sigma}(R) \in T_{\sigma(n)}^{*}(R)$ for all $R \in \mathbb{D}$.

Let $\bar{\sigma}$ be a priority function such that $\bar{\sigma}(1)=i, \bar{\sigma}(2)=j$ and $\bar{\sigma}(k)=\sigma(k)$ for all $k \neq 1,2$. We define the following SCF $f: \mathbb{R} \rightarrow A$ :

$$
f(R)= \begin{cases}f_{1}^{\sigma}(R) & \text { if } R \in \mathbb{D} \\ \text { a serial dictatorship with respect to the priority function } \bar{\sigma} & \text { if } R \notin \mathbb{D}\end{cases}
$$

We check the strategy-proofness of $f$. Again, it suffices to check that $i$ and $j$ cannot manipulate. If $R \in \mathbb{D}$, the outcome belongs to $r_{2}\left(R_{j}\right)$ i.e. $s_{j}^{k}$. Agent $j$ cannot manipulate for the following reason: for any $R_{j}^{\prime}$ if (i) $\left(R_{i}, R_{j}^{\prime} \ldots\right) \in \mathbb{D}$, then the outcome belongs to $s_{j}^{k}$ (ii) $\left(R_{i}, R_{j}^{\prime} \ldots\right) \notin \mathbb{D}$ then the outcome does not belong to $r_{1}\left(R_{j}\right)$ because $j$ is the dictator "after" $i$. Consider a possible manipulation by $i$ from $R$. Since $s_{j}^{k}$ is an isolated vertex, $s_{j}^{k} \cap r_{2}\left(R_{i}\right) \neq \emptyset$. Hence $i$ obtains a second-ranked alternative in $R_{i}$. For any $R_{i}^{\prime}$ (i) if $\left(R_{j}, R_{i}^{\prime} \ldots\right) \in \mathbb{D}$ the outcome belongs to $r_{2}\left(R_{i}^{\prime}\right)$ and $r_{1}\left(R_{i}\right) \cap r_{2}\left(R_{i}^{\prime}\right)=\emptyset$ and (ii) if $\left(R_{j}, R_{i}^{\prime} \ldots\right) \notin \mathbb{D}$ the outcome belongs to $r_{1}\left(R_{i}^{\prime}\right)$ and $r_{1}\left(R_{i}\right) \cap r_{1}\left(R_{i}^{\prime}\right)=\emptyset$. If $R \notin \mathbb{D}$, then $i$ is a dictator and cannot manipulate. For $j$, the only case to consider is the one where the manipulation $R_{j}^{\prime}$ is such that $\left(R_{j}^{\prime}, R_{i}, \ldots\right) \in \mathbb{D}$. In this case, the outcome is in $s_{j}^{k}$. At $R$ the outcome is a $R_{j}$ - maximal element in $s_{i}^{m}$. Since $s_{j}^{k}$ is an isolated vertex, it has elements in common with $s_{i}^{m}$. Hence the outcome at $R$ is weakly preferred to alternatives in $s_{j}^{k}$ accrding to $R_{j}$. Thus, $j$ cannot manipulate.

It is again straightforward to show that $f$ is unanimous and non-dictatorial.

We now prove Part B. Pick an arbitrary pair $i, j \in I$. Suppose $G\left(S_{i}, S_{j}\right)$ is not a connected graph with isolated vertices i.e. the sub-graph of $G\left(S_{i}, S_{j}\right)$ consisting of non-isolated vertices is not connected. The vertices of $G\left(S_{i}, S_{j}\right)$ can be partitioned into subsets $V^{\prime}$ and $V^{\prime \prime}$ with 
- $\left|V^{\prime}\right|,\left|V^{\prime \prime}\right| \geq 2$.

- $\{u, v\} \notin E$ for all $u \in V^{\prime}$ and $v \in V^{\prime \prime}$.

- The sub-graphs with vertices $V^{\prime}$ and $V^{\prime \prime}$ are not discrete.

For instance in Figure 4, $V^{\prime}$ is the set $\{a b c d, e f, a g, b h\}$ and $V^{\prime \prime}$ is the set $\{g h, c e, d f\}$. We now construct the same SCF as in Case I of Part A. This SCF is efficient, strategy-proof and non-dictatorial as required.

ObServation 3 The SCFs constructed in Cases II and III of Part A are not efficient. For instance in Example 3 of Case II, the outcome at $\left(R_{1}, R_{2}\right)$ (shown in Table 3$)$ is $b$ and in Example 4 of Case III, the outcome at $\left(\bar{R}_{1}, \bar{R}_{2}\right)$ (shown in Table 4 ) is $c$.

$\begin{array}{cc}R_{1} & R_{2} \\ e & c d \\ a b & b e \\ d & a \\ c & \end{array}$

Table 3: $\left(R_{1}, R_{2}\right)$

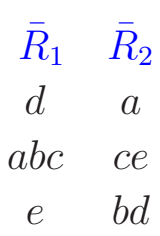

Table 4: $\left(\bar{R}_{1}, \bar{R}_{2}\right)$

Unfortunately the necessary condition in Part A of Theorem 1 is not sufficient. This is shown by the Example 8. In the next section, we will show that the condition in Part B is sufficient when there are two agents (Part D of Theorem 2).

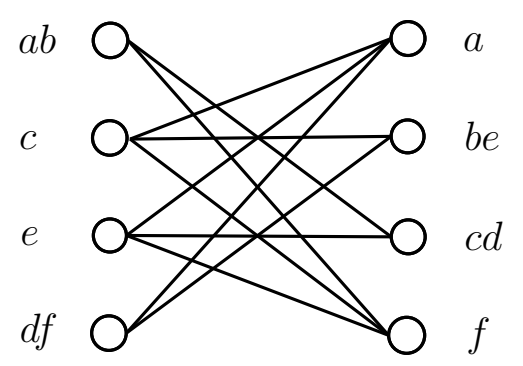

Figure 7: $G\left(S_{1}, S_{2}\right)$

Example 8 Let $I=\{1,2\}$ and $A=\{a, b, c, d, e, f\}$. Figure 7 shows the induced partition graph where $S_{1}=\{a b, c, e, d f\}$ and $S_{2}=\{a, b e, c d, f\}$. Observe that this graph is connected and each vertex has degree atleast 2 .

The SCF is described as follows: 


$$
f(R)= \begin{cases}M\left(R_{1}, r_{1}\left(R_{2}\right)\right) & \text { if } r_{1}\left(R_{2}\right) \neq a \\ M\left(R_{1},\{a, d\}\right) & \text { if } r_{1}\left(R_{2}\right)=a \text { and } r_{2}\left(R_{2}\right)=c d \\ M\left(R_{1},\{a, f\}\right) & \text { if } r_{1}\left(R_{2}\right)=a \text { and } r_{2}\left(R_{2}\right)=f \\ a & \text { if } r_{1}\left(R_{2}\right)=a, r_{2}\left(R_{2}\right)=b e, \\ & \text { and } a b \text { is preferred to } d f \text { according to } R_{1} \\ b & \text { if } r_{1}\left(R_{2}\right)=a, r_{2}\left(R_{2}\right)=b e, \\ & \text { and } d f \text { is preferred to } a b \text { according to } R_{1}\end{cases}
$$

The SCF is a serial dictatorship where 2 picks first and 1 second, for all profiles except those where the first-ranked alternative of 2 is $a$. In the latter case, the outcome specified depends on 2's second-ranked alternatives and 1's preferences. In all cases, 2 gets at least a second-ranked alternative. The only profiles that are candidates for manipulation by 2 are those where her first-ranked alternative is $a$ but the outcome is not. However, in these cases, $d f$ is preferred to $a b$ for 1 and 2 cannot obtain $a$ by misrepresentation. The outcome at all profiles is determined by maximizing 1's preferences over a set determined by 2's ordering; hence 1 cannot manipulate.

It is straightforward to verify that the SCF is non-dictatorial and satisfies unanimity.

\subsection{Sufficient Conditions for Dictatorship}

In this section we provide various sufficient conditions for dictatorship. We introduce some definitions.

Definition 7 The partition $S$ satisfies Condition $\alpha$, if (i) $v(S) \geq 2$ and (ii) there exists an agent $i$ for whom $S_{i}$ has at least three elements.

Definition 8 Let $I=\{i, j\}$. The partition $S$ satisfies Condition $\beta$ if (i) $v(S)=1$ and (ii) there exist $s_{i}^{k}, s_{i}^{r} \in S_{i}, s_{i}^{k^{\prime}}, s_{i}^{r^{\prime}} \in S_{j}$ such that $s_{i}^{k} \subset s_{j}^{k^{\prime}}, s_{j}^{r^{\prime}} \subset s_{i}^{r}$ and $s_{j}^{k^{\prime}} \cap s_{i}^{r}=\emptyset$ (the subset relations are strict).

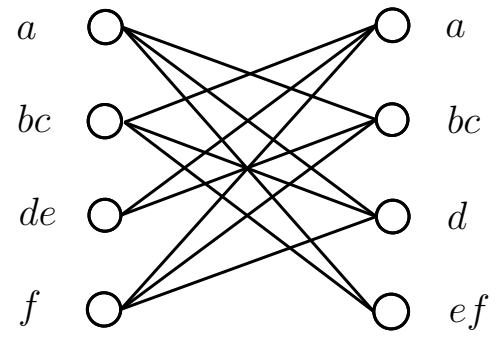

Figure 8: Partition graph for Condition $\alpha$

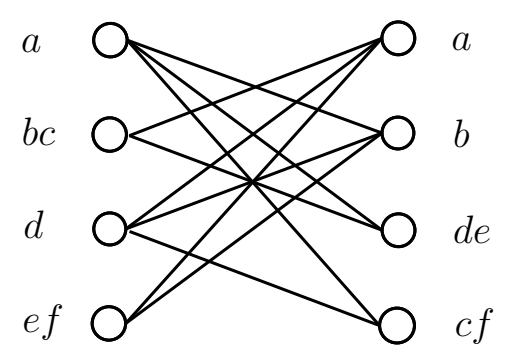

Figure 9: Partition graph for Condition $\beta$ 
OBSERVATION 4 Figure 8 shows an example of a partition graph for two players $i$ and $j$ where $S$ satisfies Condition $\alpha$ (for instance, $S_{i}=\{a, b c, d e, f\}, S_{j}=\{a, b c, d, e f\}$ and $V(S)=\{a, b c\})$. Observe that the vertices representing the elements of $V(S)$ have an edge with all vertices of the other agent (other than itself). This fact together with the assumption that $V(S) \geq 2$ ensures that the partition graph for any pair of agents is connected and the degree of every vertex is at least two.

Figure 9 illustrates Condition $\beta$ (for instance, $S_{i}=\{a, b c, d, e f\}, S_{j}=\{a, b, d e, c f\}$, $V(S)=a$ and $s_{i}^{k}, s_{i}^{r}, s_{i}^{k^{\prime}}, s_{i}^{r^{\prime}}$ are $d, b c, d e, b$ respectively). Note that the vertex representing the common element of the partition has an edge with all vertices of the other agent (other than itself). In addition, $s_{i}^{k}$ has an edge with all vertices of other agent except $s_{j}^{k^{\prime}}$. Similarly, $s_{j}^{r^{\prime}}$ has an edge with all vertices of other agent except $s_{i}^{r}$. This ensures that the graph is connected and the degree of every vertex other than $s_{j}^{k^{\prime}}$ and $s_{i}^{r}$ is at least two. The assumption that $s_{j}^{k^{\prime}} \cap s_{i}^{r}=\emptyset$ guarantees that the degree of these vertices is also at least two.

THEOREM 2 Let $S=\left(S_{1}, \ldots, S_{n}\right)$ be a partition, $n \geq 2$.

A. If $S$ satisfies Condition $\alpha$, then a strategy-proof and unanimous SCF is dictatorial.

B. If $S$ satisfies Condition $\beta$, then a strategy-proof and unanimous $S C F$ is dictatorial.

C. If $I=\{i, j\}$ and $G\left(S_{i}, S_{j}\right)$ is a cycle graph ${ }^{2}$, then a strategy-proof and unanimous $S C F$ is dictatorial.

D. If $I=\{i, j\}$ and $G\left(S_{i}, S_{j}\right)$ is a connected graph with isolated vertices, then a strategyproof and efficient $S C F$ is dictatorial.

Proof of Part A: Let $V(S)=\left\{s^{1}, s^{2}, \ldots, s^{J}\right\}$ where $J \geq 2$ and let $f: \mathbb{R} \rightarrow A$ be unanimous and strategy-proof SCF. We prove the Theorem via the following claims.

Claim 1 Let $R, R^{\prime} \in \mathbb{R}$ be such that

(i) $f(R) \in s^{j}$ where $s^{j} \in V(S)$ and

(ii) $\left\{a \in A \mid f(R) R_{i} a\right\} \subseteq\left\{a \in A \mid f(R) R_{i}^{\prime} a\right\}$ for all $i$.

Then $f\left(R^{\prime}\right) \in s^{j}$.

Proof: Pick an arbitrary agent $i$. We will show that $f\left(R_{i}^{\prime}, R_{-i}\right) \in s^{j}$. Suppose not, i.e. $f\left(R_{1}^{\prime}, R_{-1}\right) \notin s^{j}$. Clearly either $f(R) P_{i} f\left(R_{i}^{\prime}, R_{-i}\right)$ or $f\left(R_{i}^{\prime}, R_{-i}\right) P_{i} f(R)$ holds. The latter case immediately contradicts the strategy-proofness of $f$. If the former case holds, then condition (ii) above implies $f(R) P_{i}^{\prime} f\left(R_{i}^{\prime}, R_{-i}\right)$. However, $i$ will manipulate at $\left(R_{i}^{\prime}, R_{-i}\right)$ via $R_{i}$ contradicting strategy-proofness again.

\footnotetext{
${ }^{2}$ A cycle graph is a connected graph with the degree of every vertex being 2 .
} 
The Claim is established by repeated application of the same argument for different agents.

Sato (2009) proves counterparts of Claim 1 when the common indifference classes are singletons.

ClaIm 2 There exists an agent $k \in I$ for whom the follwing holds: for all $R \in \mathbb{R}, f(R) \in$ $r_{1}\left(R_{k}\right)$ whenever $r_{1}\left(R_{k}\right) \in V(S)$.

Proof: By assumption there exists $s^{j}, s^{k} \in V(S)$. Let $R^{1}$ denote the profile where $s^{j}$ and $s^{k}$ are the top and bottom indifference classes respectively for all agents. By unanimity, $f\left(R^{1}\right) \in s^{j}$.

Construct new profiles by progressively making $s^{k}$ and $s^{j}$ the best and second-best indifference classes respectively in each agent's preferenes. After changing the prefereces of all agents, the outcome must belong to $s^{k}$, by unanimity. Therefore, there must exist an agent $i$ such that (i) before agent $i$ changes his preference, the outcome belongs to $s^{j}$ and (ii) when $i$ lifts $s^{k}$ to the top of his preference, the outcome is no longer in $s^{j}$. If the outcome does not belong to either $s^{j}$ or $s^{k}$, agent $i$ will manipulate by reverting to the ordering where $s^{j}$ is first and $s^{k}$ is last and thereby obtaining an outcome in $s^{j}$. Therefore the outcome when $i$ changes her ordering, must belong to $s^{k}$. Let $R^{2}$ denote the profile where which agents 1 through $i$ have $s^{k}$ first and $s^{j}$ second. By the earlier argument $f\left(R^{2}\right) \in s^{k}$.

Next interchange $s^{j}$ and $s^{k}$ at $R_{i}^{2}$. Let $R^{3}$ denote the resulting preference profile. By stragegy-proofness, $f\left(R^{3}\right) \in s^{j}$.

At $R^{2}$ and $R^{3}$, lower $s^{j}$ to the bottom for $1,2, \ldots, i-1$ and to the second last position for $i+1, \ldots, n$. Let $R^{2^{\prime}}$ and $R^{3^{\prime}}$ denote the resulting profiles respectively. By Claim 1, $f\left(R^{2^{\prime}}\right) \in s^{k}$. In order for $i$ not to manipulate at $R^{3^{\prime}}$, we must have $f\left(R^{3^{\prime}}\right) \in\left\{s^{k} \cup s^{j}\right\}$. But if $f\left(R^{3^{\prime}}\right) \in s^{k}$, then Claim 1, implies $f\left(R^{3}\right) \in s^{k}$, which is a contradiction. Therefore $f\left(R^{3^{\prime}}\right) \in s^{j}$.

Let $d \in A \backslash\left\{s^{j} \cup s^{k}\right\}$ and let $s_{i}^{d}$ denote the indifference class of agent $i$ to which $d$ belongs. Let $R^{4}$ be the profile shown in Table 3 .

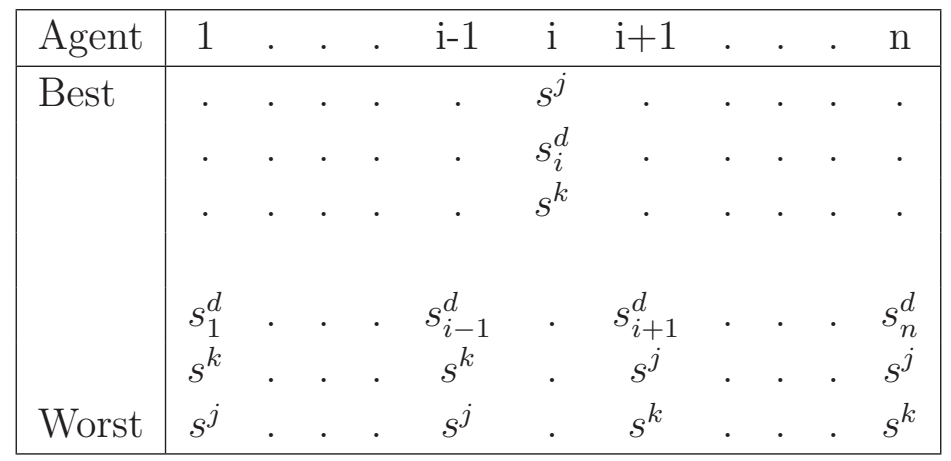

Table 5: $R^{4}$ 
Since $f\left(R^{3^{\prime}}\right) \in s^{j}$, Claim 1 implies that $f\left(R^{4}\right) \in s^{j}$. Let $R^{5}$ be the profile obtained by switching $s^{j}$ and $s^{k}$ at $R^{4}$ for agents $i+1$ through $n$. By strategy-proofness $f\left(R^{5}\right) \in\left\{s^{j} \cup s^{k}\right\}$. Suppose $f\left(R^{5}\right) \in s^{k}$. Let $R^{6}$ be the profile in Table 4. Since $f\left(R^{5}\right) \in s^{k}$, Claim 1 implies $f\left(R^{6}\right) \in s^{k}$. But this contradicts unanimity at $R^{6}$. Hence $f\left(R^{5}\right) \in s^{j}$. Since $s^{j}$ is bottom ranked for all agents other than $i$ and top ranked for $i$, it follows that the outcome belongs to $s^{j}$ whenever agent $i$ ranks $s^{j}$ first.

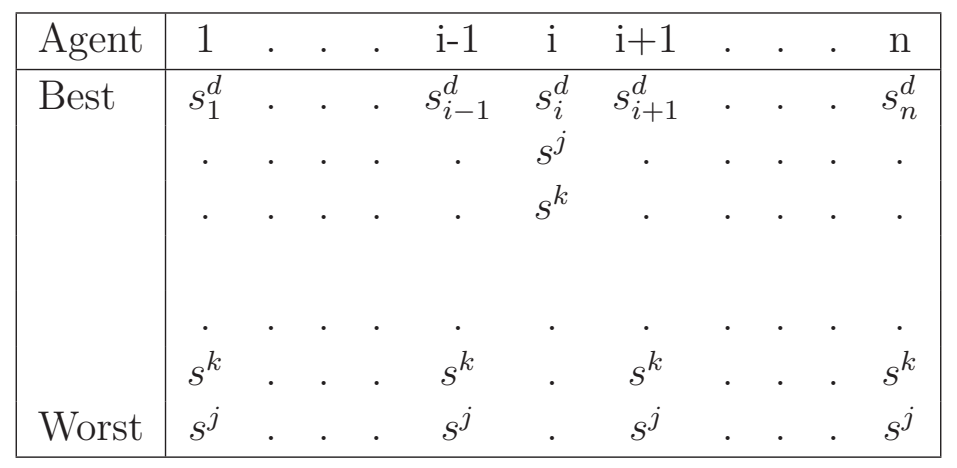

Table 6: $R^{6}$

Consider $s^{k} \in V(S)$ with $s^{k}$ distinct from $s^{j}$. The earlier arguments can be replicated to show that there exists an agent $i^{\prime}$ such that the outcome belongs to $s^{k}$ whenever $i^{\prime}$ ranks $s^{k}$ first. If $i$ and $i^{\prime}$ are distinct, the single-valuedness of $f$ is contradicted at any profile where $s^{j}$ and $s^{k}$ are ranked first by $i$ and $i^{\prime}$ respectively. This establishes the Claim.

We now complete the proof by showing that agent $i$ identified in Claim 2 is a dictator.

Suppose this is false, i.e. there exists profile $\bar{R}$ and $f(R) \notin r_{1}\left(\bar{R}_{i}\right)$. In order not to contradict Claim 2 immediately, $r_{1}\left(\bar{R}_{i}\right) \notin V(S)$. Let $s^{j}$ be the top-ranked indifference class in $V(S)$ in $\bar{R}_{i}$. By assumption there exists $s^{k} \in V(S)$ distinct from $s^{j}$. Construct profile $R^{7}$ as follows: (i) preferences of all agents other than $i$ are the same as in $\bar{R}$ and (ii) $r_{1}\left(R_{i}^{7}\right)=r_{1}\left(\bar{R}_{i}\right)$ and $r_{2}\left(R_{i}^{7}\right)=s^{k}$. By strategy-proofness $f\left(R^{7}\right) \notin r_{1}\left(R_{i}^{7}\right)$. However, $i$ can obtain an alternative in $s^{k}$ by raising $s^{k}$ to the top of her ordering (Claim 2). Hence $f\left(R^{7}\right) \in s^{k}$. Consider profiles $R^{8}$ and $R^{9}$ described in Tables 5 and 6 below.

By Claim $1 f\left(R^{8}\right) \in s^{k}$. In addition strategy-proofness and Claim 2 imply $f\left(R^{9}\right) \in s^{j}$. Pick an arbitrary alternative $d$ in $r_{1}\left(\bar{R}_{i}\right)$ and let $s_{l}^{d}$ denote the indifference class to which $d$ belongs for all agents $l \neq i$. Observe that $s_{l}^{d}$ is strictly preferred to $s^{j}$ for all $l$ in $R^{9}$. 


\begin{tabular}{|c|c|c|c|c|c|c|c|c|c|}
\hline Agent & 1 & • & . & i-1 & $\mathrm{i}$ & $i+1$ & . & - & $\mathrm{n}$ \\
\hline \multirow[t]{2}{*}{ Best } & $s^{k}$ & . & & $s^{k}$ & $r_{1}\left(\bar{R}_{i}\right)$ & $s^{k}$ & . & & $s^{k}$ \\
\hline & . & . & . & $\cdot$ & $s^{k}$ & . & . & $\cdot$ & $\cdot$ \\
\hline Worst & $s^{j}$ & . & . & $s^{j}$ & . & $s^{j}$ & . & . & $s^{j}$ \\
\hline
\end{tabular}

Table 7: $R^{8}$

\begin{tabular}{|c|c|c|c|c|c|c|c|c|c|}
\hline Agent & 1 & . & · & i-1 & i & $i+1$ & $\cdot$ & · & . $\quad n$ \\
\hline \multirow[t]{2}{*}{ Best } & $s^{k}$ & . & . & $s^{k}$ & $\bar{r}_{1}\left(\bar{R}_{i}\right)$ & $s^{k}$ & . & $\cdot$ & . $s^{k}$ \\
\hline & - & - & · & - & $s^{j}$ & - & $\cdot$ & $\cdot$ & . \\
\hline & . & . & · & . & . & . & - & · & . \\
\hline Worst & $s^{j}$ & . & . & $s^{j}$ & . & $s^{j}$ & . & . & $s^{j}$ \\
\hline
\end{tabular}

Table 8: $R^{9}$

Construct profile $R^{10}$ by raising $s_{l}^{d}$ to the top of $R_{l}^{9}$ for all $l \neq i$ while keeping $i$ 's preferences fixed at $R_{i}^{9}$. By Claim $1, f\left(R^{10}\right) \in s^{j}$. However unanimity requires $f\left(R^{10}\right) \in \bigcap_{l} r_{1}\left(R_{l}^{10}\right)$ where $d \in \cap_{l} r_{1}\left(R_{l}^{10}\right) \neq \emptyset$. We have a contradiction.

Proof of Part B: Let $S$ satisfy Condition $\beta$ and suppose $f$ is a strategy-proof and unanimous SCF defined for $I=\{i, j\}$. In order to simplify the notation, we will denote the commom indifference class by $s$, the sets $s_{i}^{k}$ and $s_{i}^{r}$ by $y$ and $X$ respectively and $s_{i}^{k^{\prime}}, s_{i}^{r^{\prime}}$ by $Y$ and $x$ respectively. Condition $\beta$ requires $y \subset Y, x \subset X$ and $X \cap Y=\emptyset$.

The proof uses the following claim.

Claim 3 For all profiles $R$ such that $r_{1}\left(R_{i}\right)=s$ and $r_{1}\left(R_{j}\right)=Y, f(R) \in s \cup Y$.

Proof: Suppose not, i.e. there exists $R^{\prime}$ with $r_{1}\left(R_{i}^{\prime}\right)=s, r_{1}\left(R_{j}^{\prime}\right)=Y$ and $f\left(R^{\prime}\right) \notin\{s \cup$ $Y$ \}. Let $R_{i}^{\prime \prime}$ and $R_{j}^{\prime \prime}$ be such that $r_{1}\left(R_{i}^{\prime \prime}\right)=s, r_{2}\left(R_{i}^{\prime \prime}\right)=y, r_{1}\left(R_{j}^{\prime \prime}\right)=Y$ and $r_{2}\left(R_{j}^{\prime \prime}\right)=s$. Strategy-proofness and unanimity imply $f\left(R_{i}^{\prime}, R_{j}^{\prime \prime}\right) \in s$ and $f\left(R_{i}^{\prime \prime}, R_{j}^{\prime \prime}\right) \in s$. However the same arguments also imply $f\left(R_{i}^{\prime \prime}, R_{j}^{\prime}\right) \in y$ and $f\left(R_{i}^{\prime \prime}, R_{j}^{\prime \prime}\right) \in Y$. This leads to a contradiction since $f$ is a function. ${ }^{3}$

Let $\bar{R}$ be such that $r_{1}\left(\bar{R}_{i}\right)=s, r_{1}\left(\bar{R}_{j}\right)=Y$. By Claim 3, $f\left(\bar{R}_{i}, \bar{R}_{j}\right) \in s \cup Y$. We complete the proof by considering the following two cases.

Case 1: $f(\bar{R}) \in s$. Using the following sequence of claims we show that $i$ is the dictator.

\footnotetext{
${ }^{3}$ These arguments closely follow counterparts in Sen (2001).
} 
ClaIm 4 For all profiles $R$ such that $r_{1}\left(R_{i}\right)=s, f(R) \in s$.

Proof: Let $R^{*}$ be such that $R_{i}^{*}=\bar{R}_{i}, r_{1}\left(R_{j}^{*}\right)=Y$ and $s$ is bottom-ranked according to $R_{j}^{*}$. If we can show $f\left(R^{*}\right) \in s$, then we are done. By Claim $3, f\left(R^{*}\right) \in s \cup Y$. Note that $f\left(R^{*}\right) \notin Y$, otherwise $j$ will manipulate at $\bar{R}$ via $R_{j}^{*}$. Therefore, $f\left(R^{*}\right) \in s$.

Claim 5 For all profiles $R$ such that $r_{1}\left(R_{i}\right)=y, f(R) \in y$.

Proof: Consider the profiles $R^{1}, R^{2}, R^{3}$ and $R^{4}$ shown in Tables 9, 10, 11 and 12 respectively. The ranking of indifference classes other than the top three is the same across the four profiles, for both agents.

\begin{tabular}{|l|cc|}
\hline Agent & $i$ & $j$ \\
\hline Best & $y$ & $x$ \\
& $s$ & $Y$ \\
& $X$ & $s$ \\
& $\cdot$ & $\cdot$ \\
Worst & $\cdot$ & $\cdot$ \\
\hline
\end{tabular}

Table 9: $R^{1}$

\begin{tabular}{|l|cc|}
\hline Agent & $i$ & $j$ \\
\hline Best & $y$ & $x$ \\
& $X$ & $Y$ \\
& $s$ & $s$ \\
& $\cdot$ & $\cdot$ \\
Worst & $\cdot$ & $\cdot$ \\
\hline
\end{tabular}

Table 10: $R^{2}$

\begin{tabular}{|l|cc|}
\hline Agent & $i$ & $j$ \\
\hline Best & $y$ & $x$ \\
& $X$ & $s$ \\
& $s$ & $Y$ \\
& $\cdot$ & $\cdot$ \\
Worst & $\cdot$ & $\cdot$ \\
\hline
\end{tabular}

Table 11: $R^{3}$

\begin{tabular}{|l|cc|}
\hline Agent & $i$ & $j$ \\
\hline Best & $y$ & $x$ \\
& $s$ & $s$ \\
& $X$ & $Y$ \\
& $\cdot$ & $\cdot$ \\
Worst & $\cdot$ & $\cdot$ \\
\hline
\end{tabular}

Table 12: $R^{4}$

We begin by showing $f\left(R^{1}\right) \in y$. If $f\left(R^{1}\right) \in A \backslash\{s \cup y\}, i$ will manipulate via an ordering where $s$ is top-ranked, thereby obtaining an alternative in $s$ (Claim 4). If $f\left(R^{1}\right) \in s, j$ will manipulate via an ordering where $Y$ is at the top obtaining an alternative in $Y$ by unanimity. Therefore $f\left(R^{1}\right) \in y$.

Strategy-proofness implies $f\left(R^{2}\right) \in y$. Our next step is to show $f\left(R^{3}\right) \in y$. If $f\left(R^{3}\right) \in$ $A \backslash\{y \cup X\}, i$ will manipulate via an ordering where $X$ is top-ranked, obtaining an alternative in $X$ by unanimity. If $f\left(R^{3}\right) \in X \backslash x$, the outcome is ranked below $Y$ by $j$ (since $X \cap Y=\emptyset$ ) and she will manipulate via an ordering where $Y$ is topranked, obtaining an alternative in $Y$ by unanimity. If $f\left(R^{3}\right) \in x, j$ will manipulate at $R^{2}$ via $R_{j}^{3}$. Therefore $f\left(R^{3}\right) \in y$. Again strategy-proofness implies $f\left(R^{4}\right) \in y$.

Finally, let $R^{5}$ be a profile such that $R_{i}^{5}=R_{i}^{4}$ and $Y$ is bottom-ranked according to $R_{j}^{5}$. We complete the proof by showing that $f\left(R^{5}\right) \in y$. If $f\left(R^{5}\right) \in A \backslash\{s \cup y\}$, then $i$ will manipulate via an ordering where $s$ is top-ranked, obtaining an alternative in $s$ by Claim 4 . If $f\left(R^{5}\right) \in s, j$ will manipulate at $R^{4}$ via $R_{j}^{5}$. Therefore $f\left(R^{5}\right) \in y$.

Claim 6 For all profiles $R$ such that $r_{1}\left(R_{i}\right)=X, f(R) \in X$.

Proof: Suppose the Claim is false, i.e. there exists a profile $\tilde{R}$ with $r_{1}\left(\tilde{R}_{i}\right)=X$ but $f(\tilde{R}) \notin X$. Let $R_{i}^{\prime \prime}$ be such that $r_{1}\left(R_{i}^{\prime \prime}\right)=X$ and $r_{2}\left({ }_{i}^{\prime \prime}\right)=y$. By strategy-proofness and Claim 
5, $f\left(R_{i}^{\prime \prime}, \tilde{R}_{j}\right) \in y$. Let $R_{j}^{\prime \prime}$ be such that top and bottom ranked indifference classes are $Y$ and $s$ respectively. Since $y \subset Y$, strategy-proofness implies that $f\left(R_{i}^{\prime \prime}, R_{j}^{\prime \prime}\right) \in Y$. Since $X \cap Y=\emptyset$, $f\left(R_{i}^{\prime \prime}, R_{j}^{\prime \prime}\right) \notin X$. Let $R_{i}^{\prime \prime \prime}$ be such that $r_{1}\left(R_{i}^{\prime \prime \prime}\right)=X$ and $r_{2}\left(R_{i}^{\prime \prime}\right)=s$. By strategy-proofness and Claim $4, f\left(R_{i}^{\prime \prime \prime}, R_{j}^{\prime \prime}\right) \in s$. If $f\left(R_{i}^{\prime \prime \prime}, R_{j}^{\prime \prime}\right) \in s$, then $j$ will manipulate via an ordering whose top-ranked indifference class has a non-empty interesection with $X$. This proves the Claim.

Claim 7 For all profiles $R, f(R) \in r_{1}\left(R_{i}\right)$.

Proof: Suppose the Claim is false, i.e. there exists a profile $R$ but $f(R) \notin r_{1}\left(R_{i}\right)$ and $r_{1}\left(R_{i}\right) \neq X, y, s$. Let $r_{1}\left(R_{i}\right)=Z$. The following cases arise.

Case 1: $Z \cap Y=\emptyset$. The arguments in Claim 6 with $Z$ substituted for $X$ can be replicated to establish $f(R) \in Z$.

Case 2: $Z \cap Y \neq \emptyset$. At $R_{j}$, lift $Y$ to the top keeping the relative ranking of other indifference classes same and let $R_{j}^{\prime}$ be the resulting ordering. By unanimity, $f\left(R_{i}, R_{j}^{\prime}\right) \in Z \cap Y$. Let $R_{i}^{\prime}$ be such that $r_{1}\left(R_{i}^{\prime}\right)=Z$ and $r_{2}\left(R_{i}^{\prime}\right)=s$. By unanimity once again, $f\left(R_{i}^{\prime}, R_{j}^{\prime}\right) \in Z \cap Y$. We will now move $Y$ downwards by progressive local switches in order to return to $R_{j}$ while arguing that the outcome remains in $Z$, thereby contradicting our initial assumption.

Suppose such a local switch does not involve $s$ and $Y$ is above $s$ in $j$ 's ranking. The outcome at such a profile cannot an alternative not in $Z \cup s$; in that case, $i$ would manipulate by raising $s$ to the top and getting $s$ (Claim 4). Nor can the outcome belong to $s$ because $j$ would manipulate via $R_{j}^{\prime}$ obtaining an alternative in $Y$. Consequently, the outcome at such a profile must belong to $Z$.

Now suppose that we reach a profile where $Y$ and $s$ are contiguous in $j$ 's ordering and $Y$ is above $s$. Let this ordering for $j$ be denoted by $\hat{R}_{j}$. By the arguments of the previous paragraph, $f\left(R_{i}^{\prime}, \hat{R}_{j}\right) \in Z$. If $f\left(R_{i}^{\prime}, \hat{R}_{j}\right) \notin Y$, then the outcome must in fact, be above $Y$ in $\hat{R}_{j}$. By switching $Y$ and $s$ in $\hat{R}_{j}$, the outcome must remain in $Z$ (by the earlier) arguments. Continuing with the switches as required, we can conclude that $f\left(R_{i}^{\prime}, R_{j}\right)$ and $f(R)$ both belong to $Z$ contradicting our assumption.

Suppose therefore that $f\left(R_{i}^{\prime}, \hat{R}_{j}\right) \in Y$, i.e. $f\left(R_{i}^{\prime}, \hat{R}_{j}\right) \in Y \cap Z$. Let $\hat{R}_{i}$ be an ordering where $Z$ and $X$ are ranked first and second respectively. By strategy-proofness, $f(\hat{R}) \in Z$. Now switch $Y$ and $s$ in $\hat{R}_{j}$. The outcome at this profile must belong to $Y \cup s$. Since $X \cap(Y \cup s)=\emptyset$, the outcome does not belong to $X$. If the outcome is ranked below $X$ in $\hat{R}_{i}, i$ will manipulate by raising $X$ to the top (Claim 6). Therefore the outcome belongs to $Z$. Now reverting back to $R_{i}^{\prime}$, we observe that the outcome remains in $Z$. Continuing with the switches in $j$ 's ordering, we conclude that $f\left(R_{i}^{\prime}, R_{j}\right)$ and $f(R)$ both belong to $Z$, once again contradicting our assumption.

We have established that $i$ is a dictator completing Case 1 .

Case 2: Suppose $f(\bar{R}) \in Y$. We will show that $j$ is the dictator. 
Claim 8 For all profiles $R$, such that $r_{1}\left(R_{j}\right)=s, f(R) \in s$.

Proof: Consider the profiles $R^{6}, R^{7}, R^{8}$ and $R^{9}$ shown in Tables 13, 14, 15 and 16 respectively. The ranking of indifference classes other than the top three is the same across the four profiles, for both agents.

\begin{tabular}{|l|cc|}
\hline Agent & $i$ & $j$ \\
\hline Best & $s$ & $Y$ \\
& $X$ & $s$ \\
& $y$ & $x$ \\
& $\cdot$ & $\cdot$ \\
Worst & $\cdot$ & $\cdot$ \\
\hline
\end{tabular}

Table 13: $R^{6}$

\begin{tabular}{|l|cc|}
\hline Agent & $i$ & $j$ \\
\hline Best & $X$ & $Y$ \\
& $s$ & $s$ \\
& $y$ & $x$ \\
& $\cdot$ & $\cdot$ \\
Worst & $\cdot$ & $\cdot$ \\
\hline
\end{tabular}

Table 14: $R^{7}$

\begin{tabular}{|l|cc|}
\hline Agent & $i$ & $j$ \\
\hline Best & $X$ & $s$ \\
& $s$ & $Y$ \\
& $y$ & $x$ \\
& $\cdot$ & $\cdot$ \\
Worst & $\cdot$ & $\cdot$ \\
\hline
\end{tabular}

Table 15: $R^{8}$

\begin{tabular}{|l|cc|}
\hline Agent & $i$ & $j$ \\
\hline Best & $X$ & $s$ \\
& $s$ & $x$ \\
& $y$ & $Y$ \\
& $\cdot$ & $\cdot$ \\
Worst & $\cdot$ & $\cdot$ \\
\hline
\end{tabular}

Table 16: $R^{9}$

By Claim 3, $f\left(R_{i}^{6}, \bar{R}_{j}\right) \in s \cup Y$. If $f\left(R_{i}^{6}, \bar{R}_{j}\right) \in s$, then $i$ will manipulate at $\bar{R}$ via $R_{i}^{6}$. Therefore $f\left(R_{i}^{6}, \bar{R}_{j}\right) \in Y$. Strategy-proofness implies $f\left(R^{6}\right) \in Y$. Strategy-proofness and $X \cap Y=\emptyset$ implies $f\left(R^{7}\right) \notin\{s \cup X\}$; otherwise $i$ will manipulate at $R^{6}$ via $R_{i}^{7}$. Unanimity implies that $f\left(R^{7}\right) \in y$. Since $y \subset Y, f\left(R^{7}\right) \in Y$. Note that strategy-proofness implies $f\left(R^{8}\right) \in s \cup Y$, because $R^{8}$ is obtained by switch $s$ and $Y$ for $j$ at $R^{7}$. However, $f\left(R^{8}\right) \in Y$ would lead to manipulation by $i$ via an ordering where $s$ is top-ranked. Hence $f\left(R^{8}\right) \in s$. By strategy-proofness, $f\left(R^{9}\right) \in s$. Let $R^{10}$ be such that $R_{j}^{10}=R_{j}^{9}, r_{1}\left(R_{i}^{10}\right)=X$ and $s$ is at the bottom according to $R_{i}^{10}$. Note that $f\left(R^{10}\right) \in x \cup s$, otherwise $j$ will manipulate via an ordering where $x$ is top-ranked, obtaining an alternative in $x$ by unanimity. But $f\left(R^{10}\right) \notin x$, otherwise $i$ would manipulate at $R^{9}$ via $R_{i}^{10}$. Therefore, $f\left(R^{10}\right) \in s$. This establishes the fact that $f(R) \in s$ for all $R$ such that $r_{1}\left(R_{j}\right)=s$.

Claims 5, 6 and 7 can now be replicated with agent $i$ replaced by $j$ to show that $j$ is the dictator.

Proof of Part $C$ : Let $G\left(S_{i}, S_{j}\right)$ be a cycle graph and suppose $f$ satisfies strategy-proofness and unanimity on this domain. In order to simplify the notation, we will denote the elements of $S_{i}$ and $S_{j}$ (i.e the vertices of $G\left(S_{i}, S_{j}\right)$ ) by $X, Y, Z, P, Q, T, \ldots$ etc.

We identify two properties of $G\left(S_{i}, S_{j}\right)$ that will be required for the argument.

Claim 9 1. $\left|S_{i}\right|=\left|S_{j}\right| \geq 3$. 2. If $(X, P)$ is an edge where $X \in S_{i}$ and $P \in S_{j}$, there exist $Y \in S_{i}, Q \in S_{j}$ such that (i) $Y \cap Q=\emptyset$ (ii) $Y \cap P \neq \emptyset$ and (iii) $X \cap Q \neq \emptyset$.

Proof: 1. $\left|S_{i}\right|=\left|S_{j}\right|$ is a standard property of bipartite cycle graphs (see page 24 of West (2001)). The only case to rule out is $\left|S_{i}\right|=\left|S_{j}\right|=2$. Suppose this is true. Let $S_{i}=\{X, Y\}$ and $S_{j}=\{P, Q\}$. Since the degree of each vertex is 2 , it must be the case that 
$(X, P),(X, Q),(Y, P)$ and $(Y, Q)$ are all edges, i.e. $X \cap P, X \cap Q, Y \cap P$ and $Y \cap Q$ are all empty. This implies $(X \cup Y) \cap(P \cup Q)=\emptyset$ which is impossible because $X \cup Y=P \cup Q=A$. 2. Let $(X, P)$ be an edge with $X \in S_{i}$ and $P \in S_{j}$. There must exist $Q \in S_{j}$ such that $X \cap Q \neq \emptyset$, i.e. $(X, Q)$ is not an edge. Since $Q$ has degree 2 , there exist vertices $Y, Z \in S_{i}$ such that $(Y, Q)$ and $(Z, Q)$ are edges. Suppose $Y \cap P$ and $Z \cap P$ are both empty. Then $P$ is degree at least three which is impossible. Hence either $Y$ or $Z$ (or both) has a non-empty intersection with $P$.

CLAIM 10 For all profiles $R$ such that $r_{1}\left(R_{i}\right) \cap r_{1}\left(R_{j}\right)=\emptyset, f(R) \in r_{1}\left(R_{i}\right) \cup r_{1}\left(R_{j}\right)$.

Proof: Pick $R$ such that $r_{1}\left(R_{i}\right)=X$ and $r_{1}\left(R_{j}\right)=P$ and $X \cap P=\emptyset$. Assume for contradiction that $f(R) \notin X \cup P$. From Claim 9 (Part 2), there exists $Y \in S_{i}, Q \in S_{j}$ such that $X \cap Q \neq \emptyset, Y \cap P \neq \emptyset$ and $Y \cap Q=\emptyset$.

Raise $Y$ to the second-ranked position in $R_{1}$ keeping the ranking of other indifference classes the same and let $R_{i}^{\prime}$ be the resulting ordering. Similarly, raise $Q$ to the second-ranked position keeping the relative ranking of other indifference classes the same and let $R_{j}^{\prime}$ be the resulting ordering. Standard strategy-proofness and unanimity arguments can be applied to show that either $f\left(R_{i}^{\prime}, R_{j}^{\prime}\right) \in X \cap Q$ or $f\left(R_{i}^{\prime}, R_{j}^{\prime}\right) \in Y \cap P$. We complete the proof of the claim by showing that $f\left(R_{i}, R_{j}\right) \in X$ if $f\left(R_{i}^{\prime}, R_{j}^{\prime}\right) \in X \cap Q$ (an analogous argument establishes $f\left(R_{i}, R_{j}\right) \in P$ if $\left.f\left(R_{i}^{\prime}, R_{j}^{\prime}\right) \in Y \cap P\right)$.

Strategy-proofness and unanimity imply $f\left(\bar{R}_{1}, \bar{R}_{2}\right) \in X$ where $r_{1}\left(\bar{R}_{1}\right)=X, r_{1}\left(\bar{R}_{2}\right)=P$ and $r_{2}\left(\bar{R}_{2}\right)=Q$. If $r_{2}\left(R_{2}\right)=r_{2}\left(\bar{R}_{2}\right)=Q$, we are done. So, let $r_{2}\left(R_{2}\right)=T \neq Q$. We consider two cases.

Case 1: $T \cap X=\emptyset$. Let $R^{\prime \prime}$ be a profile such that $r_{1}\left(R_{i}^{\prime \prime}\right)=X, r_{1}\left(R_{j}^{\prime \prime}\right)=P, r_{2}\left(R_{j}^{\prime \prime}\right)=Q$ and $r_{3}\left(R_{j}^{\prime \prime}\right)=T$. Note that $f\left(R^{\prime \prime}\right) \in X$. Since degree of $T$ is 2 , there exists $Z \in S_{i}$ such that $Z \cap T=\emptyset$. Applying Claim 9 (Part 1), $Z \cap P \neq \emptyset$. .

Suppose $Z \cap Q=\emptyset$. Raise $Z$ to the second position in $R_{i}^{\prime \prime}$ keeping the ranking of other indifference classes the same and let $R_{i}^{\prime \prime \prime}$ be the resulting ordering. By strategy-proofness, $f\left(R_{i}^{\prime \prime \prime}, R_{j}^{\prime \prime}\right) \in X$. At $R_{j}^{\prime \prime}$ switch $Q$ and $T$ and let $R_{j}^{\prime \prime \prime}$ be the resulting ordering. By strategyproofness and unanimity, $f\left(R_{i}^{\prime \prime \prime}, R_{j}^{\prime \prime \prime}\right) \in X$. If $r_{3}\left(R_{j}\right)=Q$, strategy-proofness and unanimity imply $f\left(R_{i}^{\prime \prime \prime}, R_{j}\right) \in X$; moreover $f(R) \in X$. Let $r_{3}\left(R_{j}\right)=P^{\prime} \neq Q$. By the fact that the degree of $P$ is 2 , there exists $X^{\prime} \in S_{i}$ such that $X^{\prime} \cap P^{\prime}=\emptyset$ and $X^{\prime} \cap P \neq \emptyset$. Let $\tilde{R}_{i}$ be an ordering such that $r_{1}\left(\tilde{R}_{i}\right)=X$ and $r_{2}\left(\tilde{R}_{i}\right)=X^{\prime}$. By strategy-proofness, $f\left(\tilde{R}_{i}, R_{j}^{\prime \prime \prime}\right) \in X$. Let $\tilde{R}_{j}$ be such that $r_{1}\left(\tilde{R}_{j}\right)=P, r_{2}\left(\tilde{R}_{j}\right)=T$ and $r_{3}\left(\tilde{R}_{j}\right)=P^{\prime}$. By strategy-proofness, $f\left(\tilde{R}_{i}, \tilde{R}_{j}\right) \in X$. Again by strategy-proofness, $f\left(\tilde{R}_{i}, R_{j}\right) \in X$ and $f\left(R_{i}, R_{j}\right) \in X$.

Suppose $Z \cap Q \neq \emptyset$. Since the degree of $Z$ is two, there exists $Q^{\prime} \in S_{j}$ such that $Z \cap Q^{\prime}=\emptyset$. Let $\hat{R}_{j}$ be such that $r_{1}\left(\hat{R}_{j}\right)=P, r_{2}\left(\hat{R}_{j}\right)=Q, r_{3}\left(\hat{R}_{j}\right)=T$ and $r_{4}\left(\hat{R}_{j}\right)=Q^{\prime}$. Note that $f\left(R_{i}^{\prime \prime \prime}, \hat{R}_{j}\right) \in X$. Let $R_{j}^{*}$ be such that $r_{1}\left(R_{j}^{*}\right)=X, r_{2}\left(R_{j}^{*}\right)=T, r_{3}\left(R_{j}^{*}\right)=Q^{\prime}$ and $r_{4}\left(R_{j}^{*}\right)=Q$. By strategy-proofness and unanimity, $f\left(R_{i}^{\prime \prime \prime}, R_{j}^{*}\right) \in X$. Applying these arguments repeatedly, we conclude that $f(R) \in X$. 
Case 2: $T \cap X \neq \emptyset$. Since the degree of $P$ is 2, there exists $Y^{\prime} \in S_{1}$ such that $Y^{\prime} \cap T=\emptyset$ and $Y^{\prime} \cap P \neq \emptyset$. At $R_{i}^{\prime \prime}$ lift $Y^{\prime}$ to the second ranked position keeping the relative ranking of other indifference classes same and let $R_{i}^{*}$ be the resulting ordering. By strategy-proofness and unanimity, we can infer that $f\left(R_{i}^{*}, R_{j}^{\prime \prime}\right) \in X$ and $f\left(R_{i}^{*}, R_{j}^{\prime \prime \prime}\right) \in X$. Applying these arguments repeatedly, $f(R) \in X$ follows.

The proof is completed by showing that the agent from whose first-ranked indifference class the outcome is picked in Claim 10 is in fact, a dictator. This can be done by replicating the arguments in Claim B in Sen (2001). The only requirement for the argument is for the graph $G\left(S_{i}, S_{j}\right)$ to be connected.

Proof of Part D: Let $G\left(S_{i}, S_{j}\right)$ be a connected graph with isolated vertices and suppose $f$ satisfies strategy-proofness and efficiency on this domain.

ClaIm 11 For all $R$ such that $r\left(R_{i}\right) \cap r\left(R_{j}\right)=\emptyset$, either $f(R) \in r_{1}\left(R_{i}\right)$ or $f(R) \in r_{1}\left(R_{j}\right)$.

Proof: Pick $R$ such that $r\left(R_{1}\right) \cap r\left(R_{2}\right)=\emptyset$ and denote $r_{1}\left(R_{i}\right)$ and $r_{1}\left(R_{j}\right)$ by $X$ and $P$ respectively. Suppose $f(R) \notin X \cup P$. There exists $Q \in S_{j}$ such that $X \cap Q \neq \emptyset$ and $Y \in S_{i}$ such that $Y \cap P \neq \emptyset$.

Let $R_{i}^{\prime}$ and $R_{j}^{\prime}$ be such that $r_{1}\left(R_{i}^{\prime}\right)=X, r_{2}\left(R_{i}^{\prime}\right)=Y, r_{1}\left(R_{j}^{\prime}\right)=P$ and $r_{2}\left(R_{j}^{\prime}\right)=Q$. By strategy-proofness and efficiency, $f\left(R_{i}^{\prime}, R_{j}\right) \notin Y \cap P$. By strategy-proofness, $f\left(R_{i}^{\prime}, R_{j}^{\prime}\right) \in P$. Similarly, strategy-proofness and efficiency imply that $f\left(R_{i}, R_{j}^{\prime}\right) \in X \cap Q$. Once again applying strategy-proofness we get $f\left(R_{i}^{\prime}, R_{j}^{\prime}\right) \in X$ - a contradiction.

Suppose $X \in S_{i}$ is an isolated vertex in $G\left(S_{i}, S_{j}\right)$. If $r_{1}\left(R_{i}\right)=X$ at $R$, we have $r_{1}\left(R_{i}\right) \cap$ $r_{1}\left(R_{j}\right) \neq \emptyset$. The same conclusion holds if $X$ is isolated and belongs to $S_{j}$.

Finally, since non-isolated vertices are connected in $G\left(S_{i}, S_{j}\right)$, the arguments in Claim B in Sen (2001) can be used in conjunction with Claim 11 to demonstrate the existence of a dictator.

We make several observations about our result.

ObSeRvation 5 Part A of Theorem 2 generalizes Sato (2009) in two ways. It requires only two rather three common indifference classes. Moreover, these common idifference classes need not be singletons. Our argument follows the general structure of Sato's arguments (with appropriate refinements) which in turn follows that of Reny (2001) and several other papers.

Observation 6 Parts B, C and D are two-person results. The generalization to an arbitrary number of agents is not straightforward. The main difficulty is that the standard "cloning" arguments for the situation where all agents have a common domain, cannot be used. It is not clear whether the pairwise conditions used in Parts B, C and D are sufficient for dictatorship generally. 
Observation 7 Part B of Theorem 1 together with Part D of Theorem 2 provide a necessary and sufficient condition for strategy-proofness and efficiency to imply dictatorship, in the case of two agents.

\section{Conclusion}

The paper investigates the effect of the partition structure on dictatorship results in the exogenous indifference classes model. The focus of the analysis is the pairwise partition function graph. We provide necessary conditions and stronger sufficient conditions on these graphs for strategy-proofness and unanimity (or efficiency) to imply dictatorship.

Several natural questions remain open. Some of our results apply only to the two-voter case and a gap exists between our necessary and sufficient conditions. These questions appear to be difficult but we hope to make progress in answering them in the future.

\section{REFERENCES}

Barberá, S. And L. Ehlers (2011): "Free Triples, Large Indifference Classes and the Majority Rule," Social Choice and Welfare, 37, 559-574.

Gibbard, A. (1977): "Manipulation of Voting Schemes: a General Result," Econometrica, 45, 439-446.

Hurwicz, L. (1972): "On Informationally Decentralized Systems," McGuire, B and Radner, R (eds.), Decision and Organization, Amsterdam: North-Holland Press, 297-336.

PÁpAI, S. (2000): "Strategyproof assignment by hierarchical exchange," Econometrica, 68, 1403-1433.

Reny, P. (2001): "Arrow's Theorem and the Gibbard-Satterthwaite Theorem: a Unified Approach," Economics Letters, 70, 99-105.

SATO, S. (2009): "Strategy-proof Social Choice with Exogenous Indifference Classes," Mathematical Social Sciences, 57, 48-57.

Satterthwaite, M. (1975): "Strategy-proofness and Arrow's Conditions: Existence and Correspondence Theorems for Voting Procedures and Social Welfare Functions," Journal of Economic Theory, 10, 187-217.

Satterthwaite, M. And H. Sonnenschein (1981): "Strategy-proof Allocation Mechanism at Differentiable," The Review of Economic Studies, 48, 587-597.

SEn, A. (2001): "Another Direct proof of the Gibbard-Satterthwaite Theorem," Economics Letters, 70, 381-385. 
Svensson, L.-G. (1999): "Strategy-proof Allocation of Indivisible Goods," Social Choice and Welfar, 16, 557-567.

West, D. (2001): "Introduction to Graph Theory," Prentice Hall. 